\title{
«In Afrika kann ich jeden Tag Wunder vollbringen»
}

\section{Daniel Lüthi}

Freier Journalist und Fotograf, Medientrainer, Bern

Hat dieser Mann zwei Seelen in seiner Brust? Zwei Herzen? Wie sonst ist es möglich, jahrelang zwischen zwei so verschiedenen Welten hin- und herzupendeln, und an beiden Orten zu 100 Prozent präsent und leistungsfähig zu sein? Die eine Welt ist "sein» Spital im Schweizer Jura. Und die andere «sein» Spital in Afrika. Zerrissenheit habe er noch nie gespürt, sagt Jörg Peltzer, «beides ist Teil von mir».

\section{Herr über Leben und Tod}

Hier und dort ist Peltzer Chirurg, Unfallchirurg vor allem. "Ich habe Freude am Manuellen", erklärt er, schon als Kind habe er sich besonders gerne in der Werkstatt des Vaters aufgehalten, «als Sechsjähriger konnte ich bereits gut mit einer Säge umgehen. Heute schätze ich es, dass ich Probleme mit dem Skalpell lösen kann. Eine Tablette zu verschreiben und abzuwarten, ob sie nützt, wäre nicht meine Sache.» Welcher Art die Probleme in den beiden Welten sind, zeigt die Art der Verletzungen, die es hier und dort zu behandeln gilt, oder die Liste der sehr unterschiedlichen Unfallherde: In Delémont sind es beispielsweise Motorrad- oder Reitunfälle, die die Leute ins Spital bringen. In Äthiopien Krokodil-, Hyänen- oder Schlangenbisse, Schussverletzungen, Stürze von Bäumen, schwere Verbrennungen - «und immer häufiger auch Verkehrsunfälle». Einer der grossen Unterschiede: «Die 'golden hour' gilt hierzulande als Standard. Innerhalb einer Stunde ist bei uns ein Patient in aller Regel gut versorgt. Dort kann eine einfache Schlüsselbeinfraktur zum grossen Problem werden. Unfälle sind in Afrika Grund

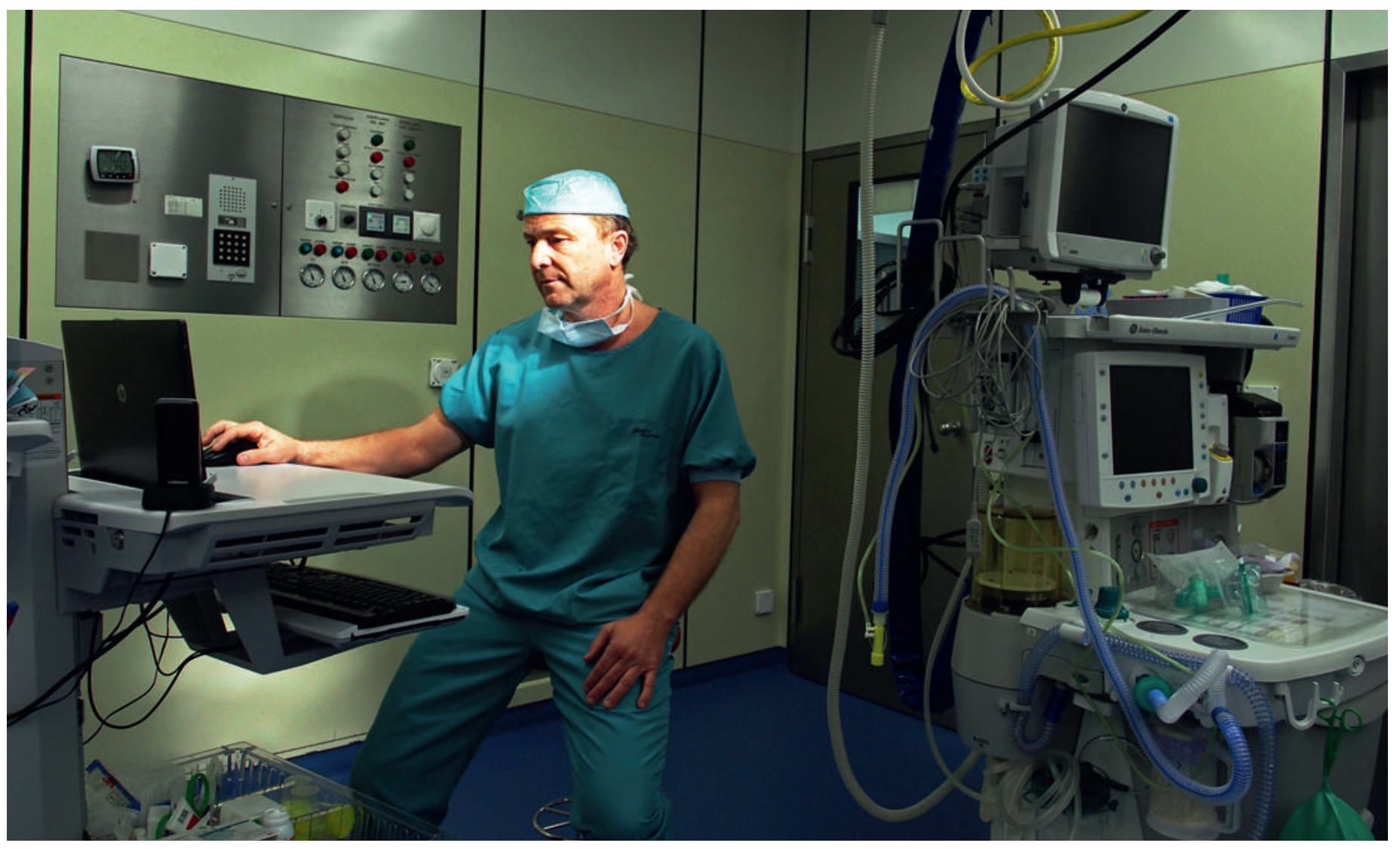


Nummer 1 für Behinderungen und Tod.» Die Patientinnen und Patienten kommen dort meistens von sehr weit her, sind oft tagelang unterwegs, bis sie endlich bei einer Klinik ankommen. "90 Prozent sterben, bevor sie ins Spital kommen, die grosse Selektion findet vorher statt», sagt Jörg Peltzer. Und dort, vor den Toren einer Institution, die Rettung verspricht, beginne der Kampf um Leben und Tod eigentlich erst recht: Nie und nimmer könnten alle behandelt werden: «Eine gute Triage gehört dort zu unseren Hauptaufgaben.» Konkret: «Ein Zwanzigjähriger hat gegenüber einem Sechzigjährigen mit ähnlichen Verletzungen Priorität. Und eine Mutter mit Kind sowieso. Grundsätzlich aber ist es etwas vom Schwierigsten, Herr über Leben und Tod zu sein und zu entscheiden, wem ich eine Chance geben soll.»

\section{Mangel an allem}

Immer zu viele Patienten also, und gleichzeitig immer zu wenig Material. Es mangle an allem: an Blutreserven, Verbandsmaterial, Instrumenten und Medikamenten. «Nicht nur Skalpell, Schere und Faden fehlen, sondern sogar Latex-Handschuhe.» Allein dies zeigt, wo in Afrika ein besonders folgenschweres Manko herrscht: bei der Hygiene. «Wir arbeiten in Operationssälen, in denen es Käfer und Fliegen gibt - und Moskitos, weil die Gitter voller Löcher sind.» Grenzwertige Erfahrun-

\section{«In Afrika ist man immer an den Grenzen.»}

gen - gerade für einen Arzt, der auch den Schweizer Standard gut kennt. «Ja», sagt Jörg Peltzer, «in Afrika ist man immer an den Grenzen.»

So schätze er den Luxus, den er in Delémont geniesse, ganz bewusst. «Hier muss ich mich nicht um die Infrastruktur kümmern, hier funktioniert alles. Das Team ist da, das Material ist da - und die Patienten sind gut versichert."

Jörg Peltzer arbeitet viel in Delémont, "80-StundenWochen mit 10 bis 12 Operationen pro Tag gibt es schon ab und zu.» Neben seiner Arbeit als Chefarzt organisiert er aber noch das Geld für die Aufbauarbeit in Äthiopien. Zurzeit betreibt seine Stiftung «Schweizer Chirurgen in Äthiopien" (www.gostar.ch) in der Stadt Jimma ein Trauma-Zentrum mit 40 Betten. «20 bis 25 Patienten können wir zusätzlich in den Gängen unterbringen.» Das Einzugsgebiet des Zentrums umfasst etwa 10 Millionen Menschen.

\section{Auslandeinsätze für Schweizer Chirurgen}

Jetzt hat die Stiftung Grosses vor: Sie plant in Jimma einen 20-Millionen-Bau mit 120 Betten und vier Ope-

\section{Jörg Peltzer}

Dr. med. Jörg Peltzer wurde 1965 in Biel geboren. Er wuchs in Biel-Benken (BaselLandschaft) auf, wo er auch die Schulen und das Gymnasium besuchte. Das Medizinstudium in Basel schloss er 1991 mit dem Staatsexamen ab. In Chur bildete er sich dann in Chirurgie und Anästhesie weiter, bevor er sechs Jahre lang für die Rega und als Chirurg in Lugano

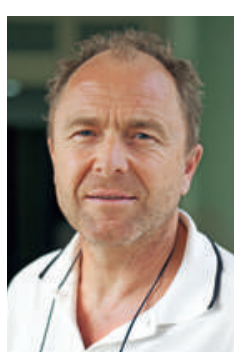
arbeitete. 1999 kam er zum ersten Mal nach Äthiopien. Ein Jahr lang arbeitete er im Spital von Metu an der Grenze zu Sudan. Zurück in der Schweiz waren die Universitätsklinik Basel und das Spitalzentrum Biel (2000/2001) seine nächsten Stationen. 2009 gründete er die Stiftung "Schweizer Chirurgen in Äthiopien" (www.gostar.ch). Diese plant jetzt ein Trauma-Zentrum in der Stadt Jimma. Seit 2003 ist Jörg Peltzer Chefarzt Chirurgie in Delémont, wo er auch wohnt.

rationssälen - «ein Trauma-Zentrum mit einer Paraplegie-Abteilung, wie es in Afrika nur wenige gibt», erläutert Jörg Peltzer hörbar stolz. Eine Schleuse soll verhindern, dass die Leute Dreck ins Spital bringen, "so schaffen wir einen neuen Hygiene-Standard». Die Operationssäle - und nur sie - seien dank Biogas- und Solaranlagen klimatisiert. "Sonst brauchen wir praktisch keinen Strom, denn auch das ist Mangelware in Äthiopien.»

Besonders wichtig bei diesem Projekt ist das Konzept, dass Schweizer Chirurgen ihre afrikanischen Kollegen vor Ort ausbilden - mit dem Ziel, dass diese das Zentrum dereinst autonom werden betreiben können. Fachkräfte im Süden abzuziehen und im Norden auszubilden sei der falsche Ansatz. "Seit 2006 waren schon rund 50 Kollegen aus der Schweiz in Jimma», sagt Jörg Peltzer, «sie alle haben erlebt, wie viel wir dort mit relativ wenig machen können. In Afrika kann ich jeden Tag Wunder vollbringen.» Etwa 100 Amputationen könnten in seiner Klinik pro Jahr dank der richtigen konservativen Behandlung vermieden werden "fantastisch, oder?».

Ein Einsatz in Afrika dauert mindestens drei Monate. Es gehe unter anderem darum, zuerst die örtlichen Sitten und Gebräuche und den anderen Rhythmus kennenzulernen, «und möglichst wenig kulturelle Fehler zu machen. Überheblichkeit ist fehl am Platz. Wir müssen Vorbilder sein und gleichzeitig bescheiden bleiben. Wir dürfen uns nicht aufführen wie die Axt im Walde - unsere Partner dürfen auf keinen Fall das Gesicht verlieren.»

Nicht nur jungen Chirurginnen und Chirurgen aus der Schweiz will Peltzer diese Erfahrungen ermöglichen, sondern neu auch solchen, die am Ende ihrer Karriere stehen. «Swiss Senior Expert Service» heisst sein jüngs- 
tes Projekt, «es richtet sich an Kolleginnen und Kollegen mit einem vollen Rucksack, die gerne noch einmal in einem ganz anderen Umfeld arbeiten würden. Afrika relativiert alles. Dort sehen sie unter anderem, wie gut es uns hier geht.»

\section{Strampeln für einen guten Zweck}

Für den Neubau in Jimma fehlen noch viele der nötigen Millionen, aber der Spiritus Rector ist zuversichtlich. Und gewohnt, dranzubleiben und zu kämpfen. Jörg Peltzer ist Velofahrer. Jeden Morgen macht er am frühen Morgen seine Runde. «Später komme ich nicht

\section{«Überheblichkeit ist fehl am Platz.»}

mehr dazu, etwas für meinen Körper zu tun, und am Abend bin ich jeweils zu müde.» Eine Fahrradtour hat auch seine Liebe zu Afrika geweckt, das war 1999. Er blieb ein Jahr, operierte schon, bevor er offiziell den entsprechenden Titel erworben hatte. "Alle sagten: Du spinnst, das ist ein Karriere-Knick», erinnert sich Peltzer mit einem Schmunzeln. "Aber es war eher ein Trampolin.» Unglaublich viel habe er damals selbständig machen können, «allgemeine und Unfall-Chirurgie, Gynäkologie - alles. Vor allem aber lernte ich, zu improvisieren.»

Etwa dreimal pro Jahr verbringt er rund zehn Tage in Äthiopien. Demnächst weiht er ein neues Personalhaus ein, das Hansjörg-Wyss-Haus. Er führt eine junge Chirurgin ein, unterschreibt Verträge mit der Universität und verhandelt mit Regierungsvertretern über legale Aspekte seines Grossprojekts.

Im November geht er in Äthiopien zum wiederholten Mal mit einer Sponsorengruppe auf Velotour. In 16 Tagen strampeln etwa 30 betuchte Europäer rund 1500 Kilometer ab, Fahrräder und Kleider werden speziell für dieses Projekt hergestellt, ein Abenteuerticket kostet 20000 Franken. «Wer dabei ist, spendet also nicht nur
Geld für unser Projekt, sondern schwitzt gleichzeitig dafür. Und erlebt hautnah, warum es nötig ist.» Der Spatenstich ist für Mitte 2017 geplant.

\section{Ein Lebenswerk}

Wenn einer beruflich so engagiert ist, ist ein Privatleben kaum mehr möglich, das sieht auch Jörg Peltzer so. «Die Frau eines engagierten Chirurgen trägt immer eine 2 auf dem Rücken.» Er selber ist geschieden, Kinder hat er keine. «Es war nie der richtige Zeitpunkt, und ich hatte Angst, ihnen nicht gerecht werden zu können.» Die Gefahr eines Burnouts streitet er keineswegs ab: «Ich versuche, mein inneres Gleichgewicht zu halten, mit Sport und dank einem tollen Freundeskreis. Und mehr als fünf Stunden Schlaf pro Nacht brauche ich glücklicherweise nicht.» In der Freizeit und während seiner eher seltenen Ferien ist er oft alleine unterwegs. "Das macht mir nichts aus", sagt er dazu. Lieber als über sein Privatleben spricht Jörg Peltzer über seine Arbeit. Vor allem diejenige in Afrika. «Etwas Komplexes schnell und schön zu machen, ist hier und dort eine Herausforderung", sagt er. "Aber hier ist vieles halt Routine.»

Wird er sich mal ganz für Afrika entscheiden? Noch brauche er die Schweiz als Basis, sagt Jörg Peltzer. «Aber wenn das neue Spital in Äthiopien mal steht, in vier oder fünf Jahren vielleicht, braucht es mich dort ganz. Das zu schaffen, wäre für mich das höchste - mein Lebenswerk.»

Bildnachweis

Fotos Daniel Lüthi

Die nächste Begegnung mit ...

Am Ende jeden Monats stellt die Schweizerische Ärztezeitung eine Persönlichkeit vor, die sich im Gesundheitswesen engagiert. Im Juni schildert Daniel Lüthi seine Begegnung mit Raphael Stolz, Allgemein- und Notfallmediziner, Notarzt am WEF und Festivalarzt des Open Airs St. Gallen. 